\title{
乙酰胆碱经人工通道的跨膜输送
}

\author{
祝平平辛鹏洋侯军利* 黎占亭* \\ (复旦大学化学系 上海 200433)
}

\begin{abstract}
摘要 乙酰胆碱具有重要的生理活性, 其跨膜输送是实现神经信号传递的重要过程之一. 利用人工合成体系来模拟乙 酰胆碱的跨膜输送过程不仅具有重要的理论研究价值, 而且还具有生物医用前景. 目前, 由于缺乏合适尺寸大小的骨 架, 构筑具有跨膜输送乙酰胆碱功能的人工通道还具有较大的挑战性. 利用含有 Phe 三肽的芳香酰朋大环化合物在脂 双层中构筑了人工跨膜通道，通过基于囊泡的动态荧光光谱实验以及基于平面脂双层的膜片针实验证实，这类人工通 道可以跨膜输送乙酰胆碱. 可以发现, 通道对乙酰胆碱的选择性随着通道孔穴尺寸的增加而增加.
\end{abstract}

关键词＼cjkstart芳香酰肼大环; 人工通道; 跨膜输送; 乙酰胆碱

\section{Transmembrane Transport of Actylcholine through Artificial Channels}

\author{
Zhu, Pingping Xin, Pengyang Hou, Jun-Li* Li, Zhan-Ting* \\ (Department of Chemistry, Fudan University, Shanghai 200433)
}

\begin{abstract}
In natural system, the transmembrane transport of actylcholine is one of the important processes for realizing the neural signal transduction, which relies on some specific proteins. The development of artificial systems displaying the similar transport capability is not only fundamentally important but also may find potential biological and medical applications. However, this has been a challenge because of lacking architectures with proper cavity size for passing of actylcholine. In this paper, the artificial channels have been built from the Phe tripeptide attached to aromatic hydrazide macrocycles. Dynamic fluorescent experiments on lipid vesicles and patch clamp experiments on planar lipid bilayer revealed that the channels can transport actylcholine. The transport selectivity was found to increase with the increasing cavity size of the macrocycles.
\end{abstract}

Keywords aromatic hydrazide macrocycles; artificial channels; transmembrane transport; acetylcholine

细胞膜的主要功能是使细胞质与外界环境分隔开 来, 从而为生命活动提供稳定的生理环境 ${ }^{[1]}$. 在细胞中, 极性的营养物质(例如阴阳离子和有机小分子)的跨膜输 送主要是依靠通道蛋白实现的. 通道蛋白在跨膜输送过 程中表现出极高的选择性与输送效率. 另外, 通道蛋白 在保持细胞内部正常的生理环境、完成生理信号的传导 和活性物质的合成等方面都有极其重要的作用 ${ }^{[2]}$. 一旦 这些蛋白功能失调, 将会引起一系列重大的疾病 ${ }^{[3]}$. 因 此, 化学家希望通过构筑人工跨膜通道来模拟通道蛋白 的结构与功能, 从而为通道病的治疗提供新的研究思 路, 也可以为研究物质跨膜输送机理提供简单模型. 三 十多年来年, 化学工作者通过不解努力发展了种类繁多 的人工跨膜通道体系 ${ }^{[4]}$. 在这些已报道的人工跨膜通道 体系中, 绝大多数是围绕离子的跨膜输送开展的. 在活
性有机小分子的跨膜输送方面, 化学家也取得了进展, 顺利实现了对糖类化合物以及氨基酸的跨膜输送 ${ }^{[5]}$. 乙 酰胆碱(actylcholine, $\mathrm{ACh}^{+}$)是一种重要的神经递质, 对 神经信号的传递发挥着重要作用. 在生物体内, 乙酰胆 碱主要由囊泡乙酰胆碱转运体(vesicular acetylcholine transporters)储存和转运 ${ }^{[6]}$. 据我们所知, 能够跨膜输送 $\mathrm{ACh}^{+}$的人工体系还未见报道, 主要是由于缺乏合适大 小的孔穴. 在本论文中, 我们报道了一类可以跨膜输送 $\mathrm{ACh}^{+}$的人工通道.

最近我们发现, 带有 Phe 三肽的芳香酰肼大环化合 物 1 2 可以高效地嵌入脂双层(图 1) $)^{[7]}$. 在嵌入脂双层 中时, 刚性的环状芳酰胇骨架位于脂双层的中间，而骨 架上的多条肽链由于受到周围磷脂分子的挤压而采取 向上或者向下的排列方式，从而使整个分子形成跨膜通

\footnotetext{
*E-mail: houj1@fudan.edu.cn; ztli@fudan.edu.cn

Received May 3, 2015; revised May 13, 2015; published online May 15, 2015.

Project supported by the National Natural Science Foundation of China (No. 21422202).

国家自然科学基金(No. 21422202 )资助项目.
} 


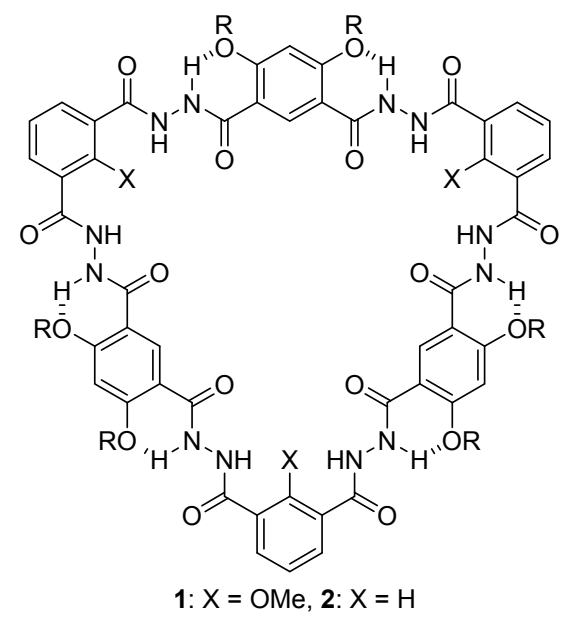

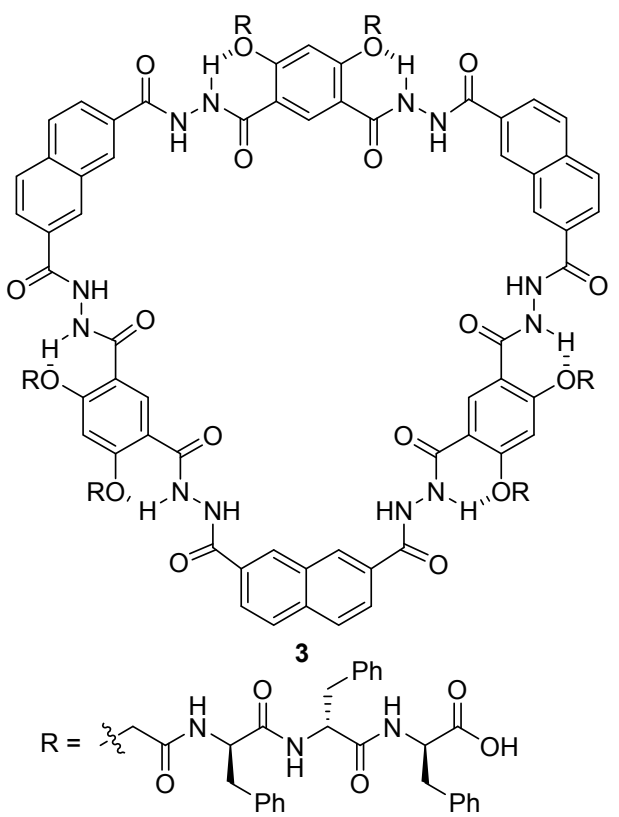

图 1 环状分子 $1 \sim 3$ 的结构

Figure 1 Structures of compounds $\mathbf{1} \sim \mathbf{3}$

道(图 2). 在之前的研究中, 这类人工跨膜通道表现出可 与天然离子通道相媲美的传输效率以及离子选择性. 由 于环状分子 1 $\sim 3$ 的刚性内穴的尺寸分别为 9.2, 9.4 和 $11.6 \AA$, 大小足以让乙酰胆碱分子通过, 并且环状骨架 上相对柔性的侧链使得环状分子 $1 \sim 3$ 在脂双层中形成 的跨膜通道内穴尺寸具有可调性 ${ }^{[7]}$. 因此, 我们推测此 类环状分子所形成的人工跨膜通道有可能实现对乙酰 胆碱的跨膜输送. 为此, 我们以磷脂分子组装形成的囊 泡和平面脂双层为平台, 通过动态苂光光谱实验和膜片 钳实验考察了环状分子 $1 \sim 3$ 对乙酰胆碱的跨膜输送性 能.

\section{1 结果与讨论}

\section{1 跨膜输送 $\mathrm{AChCl}$ 的性能研究}

在之前的研究工作中, 我们证明此类以芳酰胇为骨

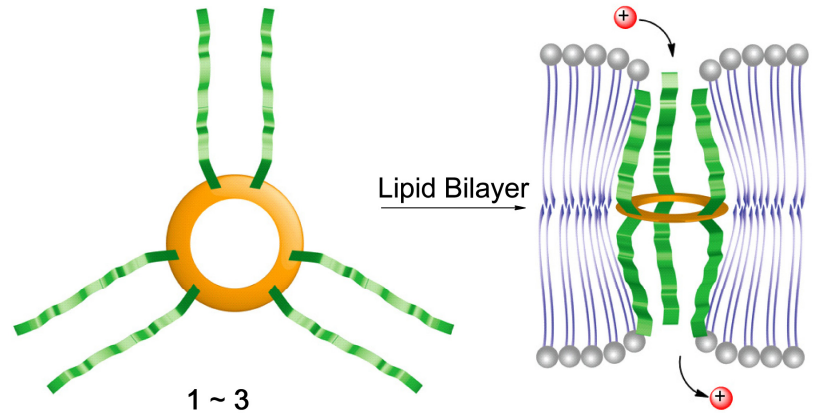

图 2 环状分子 1 3 在脂双层中形成跨膜通道示意图 Figure 2 Schematic representation for the formation of transmembrane channels from the macrocycles $\mathbf{1} \sim \mathbf{3}$ in the lipid bilayer

架的人工跨膜通道对阴阳离子的跨膜输送是以同向传 输方式进行的, 即被输送离子与带有相反电荷的离子一 起通过人工跨膜通道进入囊泡内部, 从而使囊泡内外电 荷达到平衡 ${ }^{[4]}$. 我们首先利用基于囊泡的动态荧光光谱 实验考察了化合物 $1 \sim 3$ 对氯化乙酰胆碱 $(\mathrm{AChCl})$ 的跨膜 输送性能. 考虑到 $\mathrm{ACh}^{+}$在苂光实验中不容易被检测, 所以我们通过监测 $\mathrm{Cl}^{-}$输送来考察 $\mathrm{ACh}^{+}$的跨膜输送能 力. 由于 $\mathrm{Cl}^{-}$与 $\mathrm{ACh}^{+}$是同向输送且输送速率相同, 因 此, 化合物 $\mathbf{1} \sim \mathbf{3}$ 对 $\mathrm{Cl}^{-}$输送性能就反应了它们对 $\mathrm{ACh}^{+}$ 的输送性能. 为此, 我们制备了内部包含有对 $\mathrm{Cl}^{-}$敏感 的荧光探针 Lucigenin 的囊泡. 通过在囊泡外加入 $\mathrm{AChCl}$ 并监测 Lucigenin 的荧光强度变化就能够判断 $\mathrm{AChCl}$ 是否发生了跨膜输送. 通过实验我们发现, 未加 入环状分子 1 3 时, 在 $12 \mathrm{~min}$ 内只观察到 16\%的苂光 强度变化, 说明 $\mathrm{AChCl}$ 通过自由扩散来实现跨膜输送 的能力是很弱的. 当加入 $0.1 \%$ (通道分子相对于磷脂分 子的摩尔比, 用 $x$ 表示)的环状分子 $\mathbf{1} \sim \mathbf{3}$ 时, 我们发现在 2 min 内 Lucigenin 荧光强度发生显著变化, 随后逐渐达 到平衡. 在 $12 \mathrm{~min}$ 时, 相对荧光强度分别达到 $58 \%$, 91\%和 54\%(图 3). 该实验结果说明，环状分子 1 3 均 可以促进 $\mathrm{AChCl}$ 的跨膜输送.

我们进一步对化合物 1 3 输送 $\mathrm{AChCl}$ 的性能进行 了定量的研究。考察了这些化合物在不同浓度下对 $\mathrm{AChCl}$ 输送荧光实验, 取实验开始后 $12 \mathrm{~min}$ 时的相对荧 光强度对其浓度作图(图 4). 随后用 Hill 方程拟合, 即可 得到环状分子在传输 $\mathrm{AChCl}$ 时的 $\mathrm{EC}_{50}$ (The effective concentration needed for $50 \%$ activity) 值 ${ }^{[8]} . \mathrm{EC}_{50}$ 值越小, 说明通道分子的输送效率越高. 化合物 $\mathbf{1} \sim \mathbf{3}$ 在输送 $\mathrm{AChCl}$ 时的 $\mathrm{EC}_{50}$ 值分别为: $0.024 \%, 0.017 \%$ 以及 $0.024 \%$. 这说明, 相对于环状分子 $\mathbf{1}$ 和 $\mathbf{3}$, 环状分子 $\mathbf{2}$ 在传输 $\mathrm{AChCl}$ 时表现出更高的输送效率. 化合物 $\mathbf{1}$ 和 $\mathbf{2}$ 中大环 骨架的外径尺寸相同，它们应具有相同的嵌膜能力. 但 


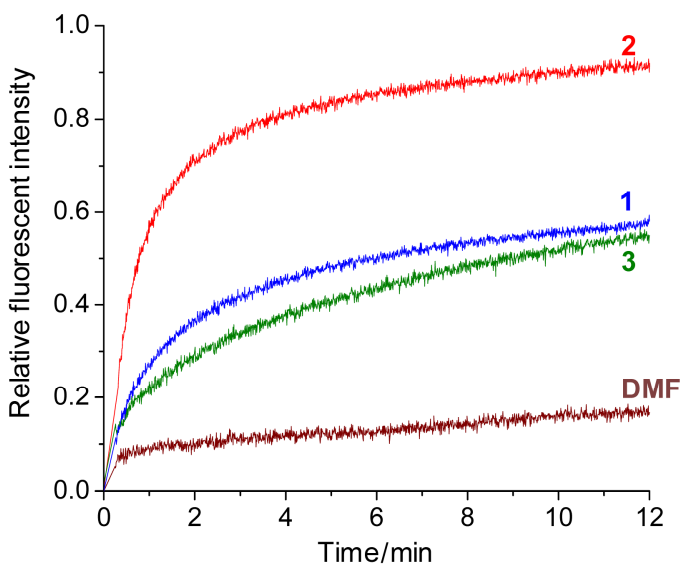

图 3 加入环状分子 $\mathbf{1} \sim 3(x=0.1 \%)$ 后囊泡中苂光素 Lucigenin $\left(\lambda_{\mathrm{ex}}=372 \mathrm{~nm}, \lambda_{\mathrm{em}}=503 \mathrm{~nm}\right)$ 相对荧光强度随时间的变化图

Figure 3 Changes in the fluorescent intensity of lucigenin $\left(\lambda_{\mathrm{ex}}=372 \mathrm{~nm}, \lambda_{\mathrm{em}}=503 \mathrm{~nm}\right)$ in vesicles with time after the addition of macrocycles $\mathbf{1} \sim \mathbf{3}(x=0.1 \%)$

是它们却具有不同的 $\mathrm{AChCl}$ 输送能力, 这可能是由于 它们结构中不同的大环内径尺寸所引起的. 相对于化合 物 1 , 化合物 $\mathbf{2}$ 中的大环结构具有更大的内径, 因此, 更 有利于具有较大体积的 $\mathrm{AChCl}$ 输送. 尽管化合物 3 中大 环的内径比 1 和 2 都要大, 但是, 这种较大面积的大环 结构导致该化合物的嵌膜能力大大降低, 从而使得它的 $\mathrm{EC}_{50}$ 值低于化合物 $\mathbf{2}$.

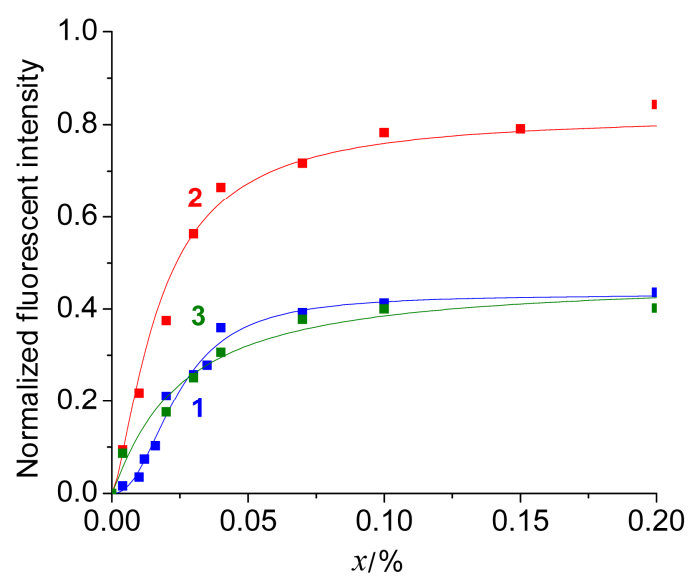

图 4 囊泡中苂光素 Lucigenin 相对荧光强度 $\left(\lambda_{\mathrm{ex}}=372 \mathrm{~nm}\right.$, $\lambda_{\mathrm{em}}=503 \mathrm{~nm}$ ) 随化合物 $1 \sim 3$ 浓度(相对于磷脂分子的摩尔比, 用 $x$ 表示)的变化图

Figure 4 Changes in normalized fluorescent intensity of Lucigenin $\left(\lambda_{\mathrm{ex}}=372 \mathrm{~nm}, \lambda_{\mathrm{em}}=503 \mathrm{~nm}\right)$ in vesicles with the concentration of $\mathbf{1} \sim \mathbf{3}$ (molar ratio relative to lipid, represented by $x$ ).

\section{2 跨膜输送 $\mathrm{AChCl}$ 的性能研究}

物质的跨膜输送可以通过 “载体” 和 “通道” 机 理进行 ${ }^{[9]}$. 通过上述荧光实验证明, 环状化合物 $\mathbf{1} \sim \mathbf{3}$ 均
可以显著增强 $\mathrm{AChCl}$ 的跨膜输送. 为了进一步研究环 状化合物跨膜输送 $\mathrm{AChCl}$ 的机理, 还在平面脂双层上 进行了膜片钳实验. 分别在脂双层膜的 trans 室加入 $\mathrm{KCl}$ 溶液 $(1.0 \mathrm{~mol} / \mathrm{L})$, 在其 $c i s$ 室加入 $\mathrm{AChCl}$ 溶液 $(1.0$ $\mathrm{mol} / \mathrm{L})$. 首先, 在不引入环状分子的前提下, 在脂双层 膜的两侧分别施加 +100 和 $-100 \mathrm{mV}$ 的电压, 通过记录 电流随时间的变化曲线, 没有观察到任何电流信号, 这 说明 $\mathrm{AChCl}$ 自身不能跨脂双层进行连续的输送. 随后, 我们在 $c i s$ 室分别加入环状分子 $\mathbf{1} \sim \mathbf{3}$ 的二甲基亚砜 (DMSO)溶液 $(0.5 \mu \mathrm{mol} / \mathrm{L})$, 搅拌 $1 \sim 2 \mathrm{~min}$ 后, 再在平面 脂双层的两侧分别施加 +100 和 $-100 \mathrm{mV}$ 的电压, 并记 录电流随时间变化曲线. 通过实验观察到了规则的方波 状电流信号(图 5), 这些方波信号说明有带电离子持续 的流过脂双层结构, 从而证实了这类环状分子在平面脂 双层中是以 “通道” 机理来实现 $\mathrm{ACh}^{+}$跨膜输送的. 另 外, 我们还发现, 在以上电流随时间变化图中, 电流信 号持续的时间可以达到 $1 \mathrm{~s}$, 明显长于其它类型的超分 子自组装通道 ${ }^{[10]}$, 说明这类通道在脂双层膜中是非常 稳定的.

我们还考察了这类通道分子对 $\mathrm{ACh}^{+}$和 $\mathrm{K}^{+}$的选择 性. 通过在脂双层膜两侧施加不同的电压, 得到了流经 该通道的电流 $(I)$ 与所施加电压 $(V)$ 之间的关系. 实验发 现，在此条件下, $I-V$ 呈现出良好的线性关系. 但由于 膜两侧的溶液不同, 导致电流与电压之间的线性关系不 经过坐标原点(图 6). 通过对 $I-V$ 图进行线性拟合就可 以得到不同环状分子在此条件下的的反转电位 $\left(V_{\mathrm{rev}}\right.$, 即 $I=0$ 时的电压值), 利用该值以及 Goldman-HodgkinKatz 方程可以计算出通道分子对 $\mathrm{ACh}^{+} / \mathrm{K}^{+}$的透过率比 值 ${ }^{[11]}$. 该比值反映了通道分子对 $\mathrm{ACh}^{+}$和 $\mathrm{K}^{+}$的相对选择 性. 通过计算得出, 环状分子 $1 \sim 3$ 对 $\mathrm{ACh}^{+} / \mathrm{K}^{+}$透过比分 别为 $0.083,0.30$ 和 0.37 . 我们可以发现, 随着环状分子 孔径的逐渐增加, 通道对 $\mathrm{ACh}^{+}$的选择性也逐渐增强. 尽管通道 $\mathbf{1}$ 比通道 $\mathbf{2}$ 的内径只增加了 $0.2 \AA$, 但对 $\mathrm{ACh}^{+}$ 选择性却明显增强, 这可能是由于它们芳香酰肼大环骨 架的差异所引起的. 在化合物 $\mathbf{2}$ 中, 骨架上缺少 3 个甲 氧基，导致该通道分子的骨架具有相对较高的柔性，使 得通道分子可以更好的与 $\mathrm{ACh}^{+}$结合，从而产生更高的 选择性. 考虑到 $\mathrm{ACh}^{+}$比 $\mathrm{K}^{+}$具有更大的体积, 在溶液中 应具有较低的迁移率. 因此, 尽管通道 2 和 3 在跨膜输 送 $\mathrm{ACh}^{+}$和 $\mathrm{K}^{+}$时优先选择 $\mathrm{K}^{+}$, 但它们仍然可以作为一 类有效输送 $\mathrm{AChCl}$ 的人工通道.

\section{2 结论}

我们通过基于囊泡的苂光光谱实验以及基于平面 脂双层的膜片钳实验，证实了以芳酰肼为骨架的环状分 

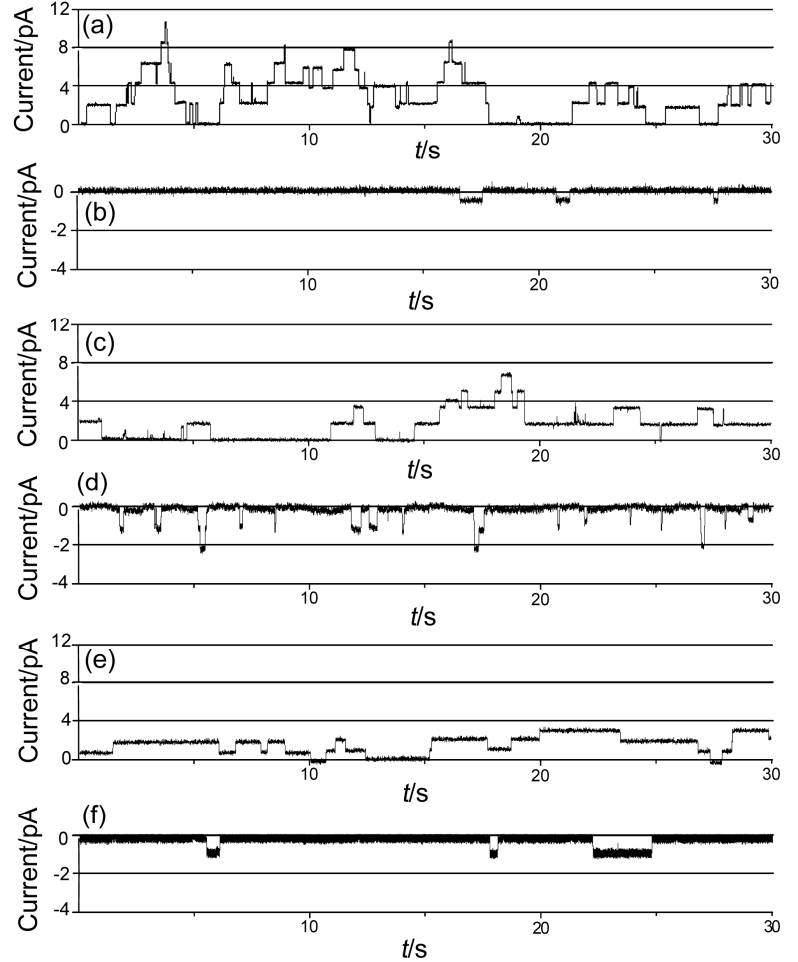

图 5 流经通道分子的电流随时间变化图

Figure 5 Current traces of the channels in the planar lipid bilayer

(a) 1 at $40 \mathrm{mV}$; (b) 1 at $-100 \mathrm{mV}$; (c) 2 at $100 \mathrm{mV}$; (d) 2 at $-100 \mathrm{mV}$; (e) $\mathbf{3}$ at $100 \mathrm{mV}$; (f) $\mathbf{3}$ at $-100 \mathrm{mV}$

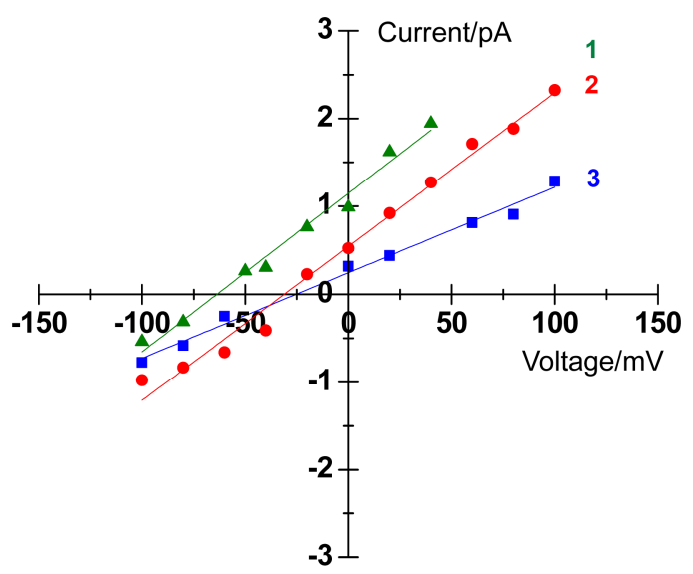

图 6 加入通道 $1 \sim 3$ 后流经通道的电流 $(I)$ 与所施加电压 $(V)$ 之 间的关系图

Figure $6 I-V$ plots for channel $\mathbf{1} \sim \mathbf{3}$ in the planar lipid bilayer in unsymmetrical solution

Trans chamber: $\mathrm{KCl}(1.0 \mathrm{~mol} / \mathrm{L})$, cis chamber: $\mathrm{AChCl}(1.0 \mathrm{~mol} / \mathrm{L})$

子可以在脂双层中形成单分子跨膜通道，并且利用这类 人工跨膜通道实现了对乙酰胆碱的跨膜输送. 由于芳酰 肼环状骨架具有广泛的官能团修饰性, 为我们进一步提 高通道分子对 $\mathrm{AChCl}$ 的选择性提供了可能.

\section{3 实验部分}

\section{1 仪器与试剂}

氯乙酰胆碱(Acetylcholine chloride)从 J\&K 公司(中 国)购得; 卵磷脂[Egg yolk $L$ - $\alpha$-phosphatidyl-choline, 氯 仿溶液 $(100 \mathrm{mg} / \mathrm{mL})$ ]从 Sigma-Aldrich 公司(美国)购得; 2-(N-吗啉代)乙磺酸(Mes)从 J\&K 公司(中国)购得; Lucigenin 从 ABBLIS 公司 (美国)购得; diPhyPC [1,2-diphytanoyl-sn-glycero-3-phosphocholine, 氯仿溶液 $(10 \mathrm{mg} / \mathrm{mL})$ ]从 Avanti Polar Lipids 公司(美国)购得. 其他 试剂未经处理直接使用.

苂光光谱用 Perkin Elmer LSS 苂光仪(USA)于室温 测得. 在膜片针实验中, 使用 Warner BC-535D 双层膜 片钳放大器来测量膜电流, 使用 PatchMaster (HEKA) 来 收集数据, 其中采样间隔为 $10 \mathrm{kHz}, 8$ 极 Bessel 滤波器的 频率为 $1 \mathrm{kHz}$ (HEKA). 使用 FitMaster (HEKA)进行数据 分析, 其中数字滤波器的频率为 $100 \mathrm{~Hz}$.

\section{2 实验方法}

\section{2 .1 囊泡的制备方法}

将卵磷脂的 $\mathrm{CHCl}_{3}$ 溶液 $(100 \mathrm{mg} / \mathrm{mL}, 0.15 \mathrm{~mL})$ 用 $\mathrm{CHCl}_{3}(5.0 \mathrm{~mL})$ 稀释后, 减压旋转蒸发成膜, 再用高真 空油原干燥 $3 \mathrm{~h}$. 取 $1.5 \mathrm{~mL}$ 含 Lucegenin 苂光素(2 $\mathrm{mmol} / \mathrm{L}$ )的缓冲液 $\left(\mathrm{pH}=6.2\right.$, Mes: $10 \mathrm{mmol} / \mathrm{L}, \mathrm{K}_{2} \mathrm{SO}_{4}$ : 70 $\mathrm{mM}$ )加入瓶中, 乳化. 使用孔径 $0.2 \mu \mathrm{m}$ 的聚碳酸酯滤膜 过滤，将得到的乳浊液放入透析袋 $(\mathrm{MWCO}=14000)$ 中, 用同样的 Mes 缓冲液透析 3 次, 尽量除尽游离于囊 泡外的苂光素, 得到包结苂光素的囊泡储备液.

\subsection{2 跨膜输送氯离子荧光实验}

在苂光比色皿中加入 $\mathrm{Mes}$ 缓冲液 $(2.0 \mathrm{~mL}, \mathrm{pH}=6.2)$ 和囊泡储备液 $100 \mu \mathrm{L}$ ，以及 $\mathrm{AChCl}(3.2 \mathrm{~mol} / \mathrm{L})$ 溶液 60 $\mu \mathrm{L}$. 通过动力学模式 $\left(\lambda_{\mathrm{ex}}=372 \mathrm{~nm}, \lambda_{\mathrm{em}}=503 \mathrm{~nm}\right)$ 监测苂 光强度变化, 待读数平稳后 $\left(I_{0}\right)$, 加入环状分子的 $\mathrm{DMF}$ 溶液, 连续监测 $12 \mathrm{~min}\left(I_{\mathrm{t}}\right)$. 最后用表面活性剂 Triton $\mathrm{X}-100$ 的水溶液 $(50 \%, 10 \mu \mathrm{L})$ 将囊泡破坏，并连续监测 至荧光强度不再变化 $\left(I_{\infty}\right)$. 将收集的数据用公式 $\left(I_{\mathrm{t}}-\right.$ $\left.I_{0}\right) /\left(I_{\infty}-I_{0}\right)$ 进行归一化处理, 得到荧光强度的变化百分 数图 ${ }^{[12]}$.

\subsection{3 基于平面脂双层的膜片钳实验}

取 $0.5 \mu \mathrm{L} 25 \mathrm{mg} / \mathrm{mL}$ diPhyPC 的癸烷储备液, 涂在 Derlin 杯(Warner Instruments, Hamden, CT) 的杯孔 $(d=$ $0.2 \mathrm{~mm}$ )周围, 先使其自然挥发, 然后用氮气流吹干. 孔 的左右两侧分别为 $c i s$ 室(接地)和 trans 室, 在 trans 室加 入 $1.0 \mathrm{~mL} 1.0 \mathrm{~mol} / \mathrm{L}$ 的 $\mathrm{KCl}$ 溶液, 在 $c i s$ 室加入 $1.0 \mathrm{~mL}$ $1.0 \mathrm{~mol} / \mathrm{L}$ 的 $\mathrm{AChCl}$ 溶液, $\mathrm{Ag}-\mathrm{AgCl}$ 电极分别插在两侧溶 液中. 接着取 $1.0 \mu \mathrm{L}$ diPhyPC 的癸烷溶液涂在之前已预 
处理过的孔上, 检测其电容, 控制电容在 $80 \sim 120 \mathrm{pF}$ 之 间 ${ }^{[13]}$. 随后通过施加电压检测其否漏电, 如不漏电即认 为小孔上已成功地形成了脂双层. 在典型的实验中, 加 入 $0.5 \mu \mathrm{L}(1.0 \mathrm{mmol} / \mathrm{L})$ 环状分子的 $\mathrm{DMSO}$ 溶液到 $c i s$ 室, 摚拌 $1 \sim 2 \min$ 后开始测试.

\section{References}

[1] Yang, F.-Y. Biomembranes, Science Press, Beijing, 2005 (in Chinese). (杨福愉, 生物膜, 科学出版社, 北京, 2005.)

[2] Hille, B. Ionic Channels of Excitable Membranes, 3rd ed, Sinauer Associates, Sunderland, MA, 2001.

[3] Ashcroft, F. M. Ion Channels and Disease, Academic Press, San Diego and London, 2000.

[4] (a) Sakai, N.; Mareda, J.; Matile, S. Acc. Chem. Res. 2005, 38, 79. (b) Li, X.; Wu, Y.-D.; Yang, D. Acc. Chem. Res. 2008, 41, 1428.

(c) Legrand, Y.-M.; Barboiu, M. Chem. Rec. 2013, 13, 524.

(d) Matle, S.; Fyles, T. M. Acc. Chem. Res. 2013, 46, 2741

(e) Gong, B.; Shao, Z. Acc. Chem. Res. 2013, 46, 2856.

(f) Montenegro, J.; Ghadiri, M. R.; Granja, J. R. Acc. Chem. Res. 2013, 46, 2955

(g) Gokel, G. W.; Murillo, O. Acc. Chem. Res. 1996, 29, 425.

(h) Mayer, M.; Yang, J. Acc. Chem. Res. 2013, 46, 2998.

(i) Xin, P.; Zhu, P.; Su, P.; Hou, J.-L.; Li, Z.-T. J. Am. Chem. Soc.
2014, 136, 13078.

[5] (a) Cho, H.; Widanapathirana, L.; Zhao, Y. J. Am. Chem. Soc. 2011, $133,141$.

(b) Hu, X.-B.; Chen, Z.; Tang, G.; Hou, J.-L.; Li, Z.-T. J. Am. Chem. Soc. 2012, 134, 8384.

(c) Chen, L.; Si, W.; Zhang, L.; Tang, G.; Li, Z.-T.; Hou, J.-L. J. Am. Chem. Soc. 2013, 135, 2152.

[6] (a) Parsons, S. M.; Prior, C.; Marshall, I. G. Int. Rev. Neurobiol. 1993, 35, 279.

(b) Usdin, T. B.; Eiden, L. E.; Bonner, T. I.; Erickson, J. D. Trends Neurosci. 1995, 18, 218.

[7] Xin, P.; Zhang, L.; Su, P.; Hou, J.-L.; Li, Z.-T. Chem. Commun. 2015, 51, 4819.

[8] Busschaert, N.; Wenzel, M.; Light, M. E.; Iglesias-Hernandez, P.; Perez-Tomas, R.; Gale, P. A. J. Am. Chem. Soc. 2011, 133, 14136.

[9] Matile, S.; Jentzsch, A. V.; Montenegro, J.; Fin, A. Chem. Soc. Rev. 2011, 40, 2453.

[10] Chui, J. K. W.; Fyles, T. M. Chem. Soc. Rev. 2012, 41, 148.

[11] Fyles, T. M.; Loock, D.; van Straaten-Nijenhuis, W. F.; Zhou, X. J. Org. Chem. 1996, 61, 8866.

[12] Jeon, Y. J.; Kim, H.; Jon, S.; Selvapalam, N.; Oh, D. Y.; Seo, I.; Park, C. S.; Jung, S. R.; Koh, D. S.; Kim, K. J. Am. Chem. Soc. 2004, 126, 15944.

[13] Ashley, R. H. In Ion Channels: a Practical Approach, Ed.: Ashley, R. H., Oxford University Press, Oxford, UK, 1995.

(Qin, X.) 